\title{
INSERÇÃO DO TEMA ATENDIMENTO INICIAL AO QUEIMADO NO CURRÍCULO DE CURSOS DE GRADUAÇÃO EM ENFERMAGEM ${ }^{1}$
}

\author{
INCLUSION OF INITIAL ASSISTANCE OF BURN VICTIMS IN THE \\ CURRICULUM FOR UNDERGRADUATE NURSING COURSES
}

\section{INSERCIÓN DEL TEMA ATENCIÓN INICIAL AL QUEMADO EN EL CURRÍCULO DE PREGRADO EN ENFERMERÍA}

\author{
William Campo Meschial ${ }^{*}$ \\ Magda Lúcia Félix de Oliveira **
}

\begin{abstract}
RESUMO
Objetivo: Verificar a inserção do tema atendimento inicial à vítima de queimadura nos currículos de cursos de graduação em Enfermagem. Método: Realizou-se um estudo documental, descritivo exploratório, de abordagem qualitativa, com análise curricular e entrevista de quatro coordenadores de cursos de Graduação em Enfermagem da região metropolitana de Maringá, Brasil, sendo os dados submetidos à análise documental e análise de conteúdo temática. Resultados: $\mathrm{O}$ atendimento inicial ao queimado está implícito no currículo de dois dos quatro cursos, porém de forma incompleta. A partir das entrevistas com os coordenadores identificou-se um "currículo oculto" desenvolvido nos cursos de graduação, porém ainda deficitário. Destacou-se a necessidade de o atendimento inicial ao queimado estar melhor estruturado nesses cursos, a importância da abordagem teórico-prática e a necessidade de adequação curricular para qualificação do ensino nesta área. Conclusão: Diante do perfil epidemiológico nacional e local, é insuficiente a abordagem dada ao atendimento inicial ao queimado na formação dos enfermeiros.
\end{abstract}

Palavras chave: Educação em enfermagem, currículo, queimaduras, enfermagem em emergência.

\begin{abstract}
Objective: To verify the inclusion of the initial care for burn victim theme to the curriculum of undergraduate nursing. Method: A documentary, exploratory descriptive study, with a qualitative approach to curriculum review was conducted as well as an interview to four course coordinators of Undergraduate Nursing in the metropolitan area of Maringa, Brazil, and the data was then subjected to document and thematic content analysis. Results: The initial assistance of burn victims is implicit in two out of four courses, but not fully covered. From the interviews with the coordinators, a "hidden curriculum" was identified, which is covered in undergraduate courses, but is still insufficient. The need for the initial assistance of burn victims to be better structured in such courses was emphasized, as well as the importance of a theoretical- practical approach and the need to adapt the curriculum to achieve teaching qualification in this area. Conclusion: It was concluded

\footnotetext{
${ }^{1}$ Artigo extraído da dissertação Atendimento Inicial ao Queimado em Unidades de Urgência: conhecimento teórico de estudantes de enfermagem, apresentada ao Programa de Pós-Graduação em Enfermagem da Universidade Estadual de Maringá (UEM), em 2012.

* Mestre. Doutorando em Enfermagem pelo Programa de Pós-Graduação em Enfermagem da UEM. Maringá, Paraná, Brasil.Email: williameschial@yahoo.com.br. Autor correspondente.

** Doutora em Saúde Coletiva. Professora Adjunto IV do Departamento de Enfermagem da UEM. Maringá, Paraná, Brasil. Email: mlfoliveira@uem.br
} 
that with regards to the national and local epidemiological profile, the approach to initial assistance of burn victims is inadequate in the undergraduate training of nurses.

Key words: Education, Nursing, curriculum, burns, emergency nursing.

\section{RESUMEN}

Objetivo: Verificar la inclusión del tema de la atención inicial a la víctima de quemaduras en los currículos de pregrado de enfermería. Material y método: Se realizó un estudio documental, de abordaje cualitativo descriptivo, exploratorio de revisión del currículo y entrevista con cuatro coordinadores de carreras de Enfermería del área metropolitana de Maringá, Brasil. Los datos fueron sometidos al análisis documental y análisis de contenido temático. Resultados: La atención inicial al quemado está implícita en el plan de estudios de dos de las cuatro carreras, sin embargo no completamente. A partir de las entrevistas con los coordinadores se identificó un "currículo oculto", desarrollado en los cursos de pregrado, sin embargo sigue siendo insuficiente. Se resaltó la necesidad de que la atención inicial al quemado esté mejor estructurada en el plan de estudios, la importancia del abordaje teórico-práctico y la necesidad de adaptarse al currículo para la cualificación de la enseñanza en esta área. Conclusión: Dado el perfil epidemiológico nacional y local, el abordaje de la atención inicial al quemado es insuficiente en la formación de enfermeros.

Palabras clave: Educación en enfermería, curriculum, quemaduras, enfermería de urgencia.

Fecha recepción: 02/25/15 Fecha aceptación: 29/03/17

\section{INTRODUÇÃO}

Acredita-se que a formação de um enfermeiro crítico-reflexivo, capaz de tomar decisões com embasamento científico em situações de urgência, como no atendimento à vítima de queimaduras, deva ser estruturada nos currículos dos cursos de Graduação em Enfermagem, os quais devem realizar ajustes na prática docente e nos projetos pedagógicos, com vistas a propiciar melhores condições de formação aos discentes.

O termo currículo deriva do latim curriculum, e possui o significado de percurso, caminho. Consiste em uma construção social, política e histórica das instituições de ensino, cuja elaboração leva em consideração saberes, práticas e experiências consideradas relevantes e associadas ao enfrentamento de problemas sociais, de maneira que relacione a formação acadêmica à realidade da sociedade (1).

No Brasil, os currículos dos cursos de en- sino superior devem atender às Diretrizes Curriculares Nacionais (DCN). Nesse sentido, desde a homologação dessas diretrizes, entre 2001 e 2004, vários esforços vêm sendo realizados para uma mudança no perfil profissional, com currículos que corroborem para a construção de um perfil acadêmico e profissional que englobe competências, habilidades e conteúdos, que confluam para novas perspectivas e abordagens formativas, que estejam de acordo com referências nacionais e internacionais e que contribua para formar profissionais resolutivos, que atuem com qualidade e eficiência, com foco nos necessidades sociais de saúde $(1,2)$.

As DCN dos cursos de Enfermagem no Brasil são estruturadas basicamente nos tópicos perfil do egresso/profissional, competências e habilidades, conteúdos curriculares, estágios e atividades complementares, organização do curso, acompanhamento e avaliação (3). Um dos pontos considerados nas DCN, para elaboração dos Projetos Pedagógicos é a adoção de conteúdos mínimos 
necessários à formação profissional (4). Esses conteúdos, apresentados por meio de eixos temáticos, devem contemplar as Ciências Biológicas e da Saúde, Ciências Humanas e Sociais e Ciências da Enfermagem, onde se incluem Fundamentos de Enfermagem, Assistência de Enfermagem, Administração de Enfermagem e Ensino de Enfermagem. Já no tópico perfil profissional, um apontamento importante diz respeito à formação de um profissional capaz conhecer e intervir sobre os problemas/situações de saúde-doença mais prevalentes no perfil epidemiológico nacional, com ênfase na sua região (4).

Diante do cenário de relevância epidemiológica das queimaduras no Brasil, considerando que atendimento às vítimas dessas injúrias é parte do cotidiano das unidades de urgência e que correspondem às principais causas externas de morbi-mortalidade (5), entende-se que o conhecimento de suas particularidades é relevante para formação de enfermeiros, visto que a adoção de medidas preventivas e curativas adequadas evita danos às vítimas e gastos para o sistema de saúde.

Dentro dessa perspectiva existe uma preocupação por parte do Ministério da Saúde com a formação, capacitação e educação permanente dos profissionais de saúde no que tange às urgências e emergências de modo geral, considerando que estas não constituem uma especialidade médica ou de enfermagem e são abordadas ainda de forma insuficiente nos cursos de graduação (6).

É relevante destacar que, segundo as DCN, preconiza-se que o enfermeiro recebe uma formação essencialmente generalista, fato que de certa forma impossibilita a formação de um profissional com capacidade para atuar de maneira integral e específica em situações de urgência e emergência, como nos atendimentos às vítimas de queimaduras. Entretanto, é pertinente que a formação integral do enfermeiro compreenda a construção de saberes, habilidades e competências necessárias à atuação frente às urgências e emergências (7).

Dessa forma, a inserção do tema atendimento inicial ao queimado (AIQ) nos currículos de Graduação em Enfermagem deve ser discutida e investigada, pois é um importante reflexo da qualidade do cuidado profissional que será dispensado ao paciente com queimaduras. Destarte, a responsabilidade de formar estudantes de enfermagem capacitados para a realidade da prática clínica é algo compartilhado entre conselhos nacionais e internacionais de enfermagem e instituições de ensino superior, sendo também uma preocupação conjunta dos Ministérios da Saúde e Educação do Brasil (8).

Diante ao exposto, questiona-se: como está contemplado o atendimento inicial ao queimado na formação acadêmica de enfermagem? Objetivou-se, então, verificar a inserção do tema atendimento inicial à vítima de queimadura, enquanto conteúdo, nos currículos de cursos de graduação em Enfermagem.

\section{MATERIAL E MÉTODO}

Trata-se de estudo documental, descritivo exploratório, de abordagem qualitativa, com entrevista de quatro coordenadores de cursos de graduação em Enfermagem e análise dos currículos dos cursos. Está inserido em um projeto de dimensão maior que buscou analisar o conhecimento de estudantes de graduação em Enfermagem sobre o atendimento inicial ao queimado em unidades de urgência.

O estudo foi realizado em Instituições de Ensino Superior (IES) da região metropolitana de Maringá (RMM) que está localizada no estado do Paraná do Brasil e conta atualmente com 26 municípios e uma população superior a 700 mil habitantes. Apenas três municípios ofertam o Curso de Graduação em Enfermagem: Maringá, Ivatuba e Manda- 
guari, que estão localizados na mesorregião Norte Central Paranaense e possuem uma população de $357.117,3.008$ e 32.658 habitantes, respectivamente (9).

O município de Maringá conta com 11 IES, em situação ativa, com cadastro no Ministério da Educação. Destas instituições, quatro ofertam o curso de graduação em Enfermagem. Ivatuba e Mandaguari contam com uma única IES em cada município nas quais o curso de graduação em enfermagem é ofertado na modalidade presencial.

Adotou-se como critério de inclusão, IES de caráter público ou privado que ofertasse o curso de Enfermagem na modalidade presencial com turmas cursando o último semestre do curso. Das quatro IES selecionadas, três estão localizadas no município de Maringá e uma no município de Ivatuba, sendo que os estágios curriculares do curso de Graduação em Enfermagem dessa IES são realizados em Maringá devido à ausência de campo para a prática no município.

A caracterização dos cursos que atenderam aos critérios de inclusão foi realizada inicialmente através do sistema eletrônico e-MEC (http://emec.mec.gov.br/), que realiza o acompanhamento dos processos que regulam a educação superior no Brasil (10). Posteriormente, essas informações foram confirmadas e complementadas na entrevista com a Coordenação dos cursos (Tabela 1).

Tabela 1. Caracterização das Instituições de Ensino Superior e Cursos de Graduação em Enfermagem da região metropolitana de Maringá que atenderam aos critérios de inclusão do estudo. Maringá - Paraná, 2012.

Instituição de Ensino Superior

\begin{tabular}{lcccc}
\hline & \multicolumn{4}{c}{ Instituição de Ensino Superior } \\
\cline { 2 - 5 } Categoria administrativa & A & B & C & D \\
\hline Privada c/ & $\begin{array}{c}\text { Privada s/ } \\
\text { fins lucrativos }\end{array}$ & $\begin{array}{c}\text { Privada c/ } \\
\text { fins lucrativos }\end{array}$ & $\begin{array}{c}\text { Pública } \\
\text { estadual }\end{array}$ \\
\hline Curação do curso & 4 anos & 4 anos & 4 anos & 4 anos \\
Carga horária do curso & 4000 & 4509 & 4189 & 4821 \\
Turmas no último semestre & 2 & 1 & 1 & 1 \\
Turno do curso & Matutino/ noturno & Matutino & Noturno & Integral \\
Vagas/ano & 175 & 40 & 100 & 44 \\
\hline
\end{tabular}

A grade curricular de cada curso foi utilizada para localizar a série e a disciplina em que é abordado o conteúdo Queimaduras, no qual poderia estar inserido o tema Atendimento Inicial ao Queimado (AIQ); e o conteúdo programático da disciplina, a carga horária e forma de avaliação.

O levantamento dos dados do currículo deu-se através da análise documental e ocorreu após a autorização e liberação pela Reitoria/Direção dos cursos de graduação das respectivas IES. Para isso, foi agendada entrevista com o coordenador do Curso de Graduação em enfermagem na própria instituição, em um único encontro, conforme disponibilidade do coordenador. Foram entrevistadas as coordenadoras de cada IES, no total de quatro, no período de março a abril de 2013. A realização da entrevista foi efetivada com o coordenador individualmente, em local privativo e sem tempo pré-estabelecido. Para a averiguação da abordagem dada ao AIQ que pudesse não estar explicitada nos currículos dos cursos. Procedeu-se a entre- 
ga do Formulário de Caracterização da IES e do Curso de Graduação em Enfermagem, sendo permitida a consulta em documentos específicos para o preenchimento do instrumento, caso o fosse necessário. Para a coleta dos dados primários, utilizou-se um roteiro de entrevista semiestruturada constituído por duas partes: caracterização do coordenador de curso; e a segunda parte constituída por três questões norteadoras, para verificar a abordagem dada ao conteúdo de queimaduras no curso de graduação. As questões foram lidas pelo pesquisador e as entrevistas, com duração média de $20 \mathrm{~min}$, foram gravadas utilizando-se gravador digital e, posteriormente, transcritas na íntegra para maior fidedignidade dos dados.

A análise dos dados seguiu os encaminhamentos da análise de conteúdo na modalidade temática, que consiste em descobrir os núcleos de sentido que compõem uma comunicação, cuja presença ou frequência tenha algum significado para o objetivo analítico visado, e, operacionalmente, abrange as fases de pré-análise, exploração do material, tratamento dos resultados obtidos e interpretação (11). Para tanto, os registros foram lidos e avaliados, seguidos da organização em três núcleos temáticos: "O paciente vítima de queimaduras e a necessidade do cuidado de enfermagem estruturado na graduação"; "O Atendimento inicial ao queimado nos currículos dos cursos: interface entre teoria e prática"; e "Necessidade de adequação curricular para qualificação do ensino em Enfermagem".

A pesquisa foi realizada após prévia autorização da Direção das IES e aprovação pelo Comitê de Ética em Pesquisa Envolvendo Seres Humanos da Universidade Estadual de Maringá (COPEP/ UEM) com parecer $n^{\circ}$ $160.458 / 2012$. Todas as diretrizes e normas regulamentadoras para pesquisa com seres humanos foram cumpridas e os coordenadores de curso assinaram o termo de consentimento anteriormente às entrevistas.
Para garantia do anonimato, as IES foram aleatoriamente nomeadas como instituições $\mathrm{A}, \mathrm{B}, \mathrm{C}$ e D, e os coordenadores foram identificados com a letra C, seguida da IES a qual pertencia.

\section{RESULTADOS}

Os resultados dos dados são apresentados em dois momentos: inserção explícita do atendimento inicial à vítima de queimaduras no currículo dos cursos, e abordagem desse tema nos currículos a partir da palavra do coordenador de curso.

\section{Assistência de enfermagem no atendi- mento inicial ao queimado e os currículos de graduação}

Após a localização das disciplinas na grade curricular e a leitura na íntegra do conteúdo programático nos currículos dos quatro cursos de graduação em enfermagem, foram localizadas palavras-chave (queimaduras; grande queimado; paciente queimado) que indicassem a inserção do conteúdo Queimaduras no componente curricular, em destaque no Quadro 1.

No curso de enfermagem da IES C este conteúdo foi identificado em duas disciplinas e em apenas uma nos cursos das demais instituições. A carga horária total das disciplinas que abordaram este conteúdo variou de 38 a $221 \mathrm{~h} /$ aula, não sendo possível identificar, a partir da análise curricular, a carga horária destinada ao conteúdo Queimaduras.

Ao analisar a ementa e os objetivos gerais e específicos de cada disciplina que abordou o conteúdo Queimaduras, constatou-se que apenas duas destacam a assistência de enfermagem a pacientes críticos, podendo-se inferir que foram abordados aspectos relativos ao atendimento inicial à vítima de queimaduras, conforme apresentado abaixo: 
Quadro 1. Inserção do tema queimaduras nos currículos de cursos de Graduação em Enfermagem da região metropolitana de Maringá. Maringá - PR, 2013.

\begin{tabular}{|c|c|}
\hline \multicolumn{2}{|r|}{ INSTITUIÇÃO DE ENSINO SUPERIOR“A” } \\
\hline Conteúdo programático: & Acidentes mais comuns na infância: queimaduras \\
\hline Disciplina: & Saúde da Criança e do Adolescente I \\
\hline Série: & Segunda \\
\hline Carga horária: & 80 h/aula \\
\hline Forma de avaliação: & Avaliação teórica, relatórios, avaliação prática e seminários \\
\hline \multicolumn{2}{|r|}{ INSTITUIÇÃO DE ENSINO SUPERIOR“B” } \\
\hline Conteúdo programático: & Situações de emergência e urgência: queimaduras \\
\hline Disciplina: & Urgência Pré-hospitalar \\
\hline Série: & Segunda \\
\hline Carga horária: & $38 \mathrm{~h} / \mathrm{aula}$ \\
\hline Forma de avaliação: & Não consta \\
\hline \multicolumn{2}{|r|}{ INSTITUIÇÃO DE ENSINO SUPERIOR “C” } \\
\hline Conteúdo programático: & Assistência ao grande queimado \\
\hline Disciplina: & Atenção aos Clientes de Alto Risco \\
\hline Série: & Terceira \\
\hline Carga horária: & 80 h/aula \\
\hline Forma de avaliação: & Avaliação teórica, estudos de caso, resumos e resenhas \\
\hline Conteúdo programático: & Recomendações nutricionais para pacientes queimados \\
\hline Disciplina: & Nutrição \\
\hline Série: & Segunda \\
\hline Carga horária: & $40 \mathrm{~h} /$ aula \\
\hline Forma de avaliação: & Avaliação teórica, avaliação prática, estudos de caso \\
\hline \multicolumn{2}{|r|}{ INSTITUIÇÃO DE ENSINO SUPERIOR“D” } \\
\hline Conteúdo programático: & Queimaduras e o cuidado de enfermagem \\
\hline Disciplina: & Cuidado de Enfermagem ao Indivíduo Adulto e Idoso \\
\hline Série: & Terceira \\
\hline Carga horária: & $221 \mathrm{~h} / \mathrm{aula}$ \\
\hline Forma de avaliação: & Avaliação teórica, prática, seminários e estudos de caso \\
\hline
\end{tabular}

IES B - Urgência Pré-hospitalar:

Ações imediatas e mediatas em situações de urgência. [...] atuar com eficiência nas situações de emergências e urgências.

IES C - Atenção aos clientes de alto risco:

Reconhecer o indivíduo em iminente gravidade $e$ prestar assistência de enfermagem integral $e$ livre de riscos [...] em Unidade de Terapia Intensiva e Pronto Socorro, promovendo a recuperação e a prevenção de sequelas. Sistematizar a assistência de enfermagem no atendimento a pacientes graves. Oferecer ao acadêmico de enfermagem as bases técnico-científicas para avaliar, reconhecer e intervir na assistência ao ser humano em risco iminente de morte.

$\mathrm{Na}$ análise das demais disciplinas, obser- 
vou-se que embora incluíssem o termo Queimaduras em seus conteúdos programáticos, não ficou evidenciada a abordagem do AIQ ao se realizar uma análise completa do programa de cada uma delas.

\section{A abordagem do conteúdo queimaduras nos currículos a partir da palavra do coor- denador de curso}

O grupo entrevistado foi composto em sua totalidade por enfermeiras, que se auto-declararam da cor branca, a maioria casadas, com idade entre 36 e 52 anos, sendo uma doutora e três mestres. O tempo de formação em Enfermagem variou de 17 a 30 anos, com média de 26 anos e o tempo na função de coordenadora do curso de Enfermagem na instituição pesquisada variou de um a 12 anos, com média de cinco anos e meio.

Foi possível identificar, através da fala das coordenadoras, outras disciplinas da grade curricular que abordaram o conteúdo Queimaduras, sem estarem explícitas no currículo dos cursos. Dessa forma, além das disciplinas apresentadas no Quadro 2, foram informadas as disciplinas: Unidade de Terapia Intensiva (IES A); Saúde da Criança (IES C); Fundamentos de Enfermagem (IES D) e Saúde do Adulto em todos os cursos analisados.

Além disso, foi unânime entre as coordenadoras de curso, que o tema Atendimento Inicial ao Queimado, de forma mais ampla e efetiva, era desenvolvido na terceira série do curso de Enfermagem, na disciplina de Saúde do Adulto e Idoso, para todos os cursos.

Ressalta-se ainda que a abordagem a este conteúdo é realizada tanto na teoria quanto na prática, com exceção à IES B, cuja coordenadora informou que o campo onde se realizam as atividades de prática clínica do curso não atende pacientes com estes agravos, ficando o aluno impossibilitado de realizar atividades práticas.

A seguir são apresentados os três núcleos temáticos provenientes das entrevistas das coordenadoras de curso:

\subsection{O paciente vítima de queimaduras e a necessidade do cuidado de enfermagem es- truturado na graduação}

Diante do cenário atual dos acidentes por queimaduras, as coordenadoras têm ciência da importância desse conteúdo na graduação de enfermagem, tendo em vista a necessidade de cuidados apresentada por esses pacientes. Dessa forma este conteúdo deve estar estruturado nos currículos dos Cursos de Graduação de Enfermagem, fornecendo ao futuro profissional elementos que possam subsidiar sua prática clínica.

O conteúdo é importante a partir do momento que te fornece subsidios para o teu atendimento ao paciente, principalmente ao grande queimado. [...] Então se você tem um profissional que não tenha conhecimento pelo menos do básico de queimaduras ele fica com dificuldade nesse atendimento $(C-I E S A)$.

Nós estamos falando de uma questão extremamente importante, relevante na formação do profissional enfermeiro, especialmente porque é um profissional que integra ativamente as equipes que prestam apoio à vítima de queimadura. [...] Logo, o preparo profissional, técnico e científico é de suma importância pra qualidade da assistência prestada pra essas vitimas (C - IES D).

Os coordenadores mostraram-se ainda cientes da relevância epidemiológica das queimaduras, visto que este tipo de injúria possui expressivos índices de morbi-mortalidade tanto nacionalmente como na região em estudo

Eu acho extremamente importante, pois podem ocorrer devido a acidentes ou até mesmo outros casos como tentativas de suicídio e homicídio (C-IES B).

Ah é um conteúdo importantíssimo por que infelizmente as queimaduras são muito frequen- 
tes. [...] Têm muitos casos de crianças queimadas com água fervendo e álcool, né (C-IES C).

\subsection{O Atendimento inicial ao queimado nos currículos dos cursos: interface entre teoria e prática}

Nesse núcleo temático as coordenadoras de curso reconhecem a importância da abordagem teórico-prática do atendimento inicial às vítimas de queimaduras, considerando ambas fundamentais e complementares. $\mathrm{O}$ conhecimento teórico, fornecido durante a graduação, é visto como um subsídio essencial a atuação do enfermeiro frente a um paciente vítima de queimaduras. Como representado na fala da coordenadora da IES A:

Se você não tiver nenhum embasamento teórico na graduação quando você for pra prática você não consegue prestar esse atendimento. [...] $O$ recém formado, se ele tiver o embasamento teórico ele consegue estar desenvolvendo as suas atividades $(C-I E S A)$.

Nesse sentido, o campo de práticas clínicas é visto pelos coordenadores como um local onde ocorre a efetiva associação entre teoria e prática. Além disso, esse ambiente permite ao estudante de Enfermagem uma vivência mais aprofundada com as questões que envolvem o cuidado humano. A abordagem prática, no entanto, nem sempre é possível de ser concretizada durantes as práticas curriculares específicas, dessa forma os docentes utilizam-se das oportunidades surgidas durante a prática clínica, mesmo que de outras áreas, para abordar a temática e inserir o aluno no cuidado.

A partir do segundo ano o aluno já vai pra campo né com práticas clínicas, supervisionado pelo professor. A partir de então ele tem uma vivência mais aprofundada com as questões que dizem respeito ao cuidado humano. [...] Nas atividades em campo de prática é que se agrupam as questões da teoria e da prática e essa prática entende-se como vivência (C-IES D).

Ele [o paciente vítima de queimaduras] pode aparecer, como eu falei, de repente quando o aluno está na prática, né lá no hospital. No segundo ano eles já fazem estágio, se tiver algum paciente queimado que dê para atender o professor já atende também. [...] O que aparece em estágio ele [o professor] já vai trabalhando mesmo que seja um conteúdo que só será dado no terceiro ano, né (C-IES C).

Logo, é preocupante o fato de que em uma das instituições não são realizadas atividades práticas de atendimento às vítimas de queimaduras, conforme referido pela coordenadora:

A prática, pelo menos eu não conheço em Maringá um ambiente onde se faça um atendimento, onde a gente vai fazer estágio, não tem um serviço específico pra atendimento de queimados. Então, eu sei que na prática tanto das clínicas quanto do estágio supervisionado pouco o aluno tem visto isso aqui em Maringá ( $C$ - IES B).

\subsection{Necessidade de adequação curricular para qualificação do ensino em Enferma- gem}

Visto que o atendimento inicial à vítima de queimaduras é realizado, na maioria das vezes, em unidades de urgência os profissionais desses serviços devem estar preparados para o atendimento dessa clientela, sendo função das entidades formadoras empoderá-los para atuar frente a esses eventos. A fala das coordenadoras de curso, no entanto, demonstra a insuficiência da abordagem do conteúdo no currículo dos cursos.

Sendo bem sincera eu acho que é um conteúdo que é pouco abordado. [...] Ele não tá explícito dentro dos programas das disciplinas, ele poderia tá um pouco mais explícito de um modo geral acho que ele tá de uma maneira implícita 
dentro dos conteúdos (C-IES B).

[...] não tem nenhuma disciplina específica pra queimado, o conteúdo fica dentro da disciplina de Saúde do Adulto, Unidade de Terapia Intensiva e dentro da disciplina de Saúde da Criança, mas de uma forma pouco pronunciada (C $-I E S)$.

Porém, a fala das coordenadoras demonstra a preocupação das mesmas com a abordagem dada ao AIQ e também a necessidade de adequação curricular:

Ele [o aluno] pode receber esse cliente às vezes até num hospital geral mesmo né, mesmo ele estando fora de um serviço especializado. Então a gente precisa pra esse novo currículo agora a gente precisa melhorar isso, nós podemos melhorar (C-IES B).

Nós trabalhamos com um projeto pedagógico que integram eixos né, [...] em que o cuidado humano perpassa todas as séries. [...] Então se pensarmos na questão da vítima de queimaduras como um paciente que requer esse cuidado, teoricamente, pelo nosso projeto, nós deveríamos estar percorrendo todas as séries. Ainda né numa tentativa de grande desafio, nós assumimos a nossa limitação de que isso na verdade na prática tem acontecido ainda de uma forma tímida né $(C-I E S D)$.

\section{DISCUSSÃO E CONCLUSÃO}

A análise documental permitiu a identificação de algumas lacunas nos currículos dos cursos em relação ao tema pesquisado. No currículo da IES A, a assistência de enfermagem a pacientes queimados é restrita às crianças e adolescentes e não há enfoque no atendimento inicial. A IES B, embora contemple o AIQ no atendimento pré-hospitalar, este está inserido em uma disciplina de carga horária pouco expressiva, e a aborda- gem é parcial, uma vez que a sequência dos cuidados ao paciente queimado é realizada intra-hospitalar e, além disso, a disciplina não descreve as formas de avaliação do aluno. A IES $C$ apresentou uma abordagem ao AIQ mais satisfatória em relação às demais, uma vez que aborda a assistência de enfermagem ao paciente grande queimado em uma disciplina de cuidados críticos e aspectos relacionados à sua nutrição em disciplina específica. Na IES D, o conteúdo insere-se em uma disciplina de cuidados de enfermagem a pacientes adultos e idosos, porém sem ênfase na atenção a pacientes críticos.

$\mathrm{O}$ atendimento inicial ao queimado segue as mesmas premissas indicadas a vítimas de trauma e quando realizado de forma adequada é capaz de reduzir a gravidade e a profundidade da lesão, refletindo em melhores resultados na recuperação dos pacientes, que incluem redução no tempo de hospitalização e na realização de procedimentos cirúrgicos (12).

Dessa forma, para que o paciente em estado crítico, como aquele vítima de queimaduras, não seja sub-assistido, a formação do profissional de enfermagem deve contemplar os conteúdos relacionados ao desequilíbrio das funções orgânicas, mas também estratégias que facilitem o desenvolvimento de competências e habilidades, que possibilitem, em nível prático, a avaliação sistemática e completa do paciente. Busca-se assim o reconhecimento de estados clínicos atuais ou potenciais de deterioração, a realização de intervenções resolutivas precocemente, avaliação das repostas do paciente e a identificação de materiais e recursos indicados a cada situação (13).

Nesse sentido merece destaque o estágio curricular supervisionado que é efetivado nos dois últimos semestres dos cursos, porém este não foi mencionado pelas coordenadoras como disciplina em que estivesse incluso o atendimento à vítima de queimaduras. Segundo as diretrizes curriculares dos cursos de graduação em enfermagem, estes 
devem obrigatoriamente incluir além dos conteúdos teóricos e práticos, o estágio curricular supervisionado, que deverá ocorrer em hospitais gerais e especializados, ambulatórios, rede básica de serviços de saúde e comunidades, nos dois últimos semestres do curso (14).

A partir do estágio curricular supervisionado, o estudante de enfermagem tem uma visão de forma ampla e concreta de sua futura profissão. Além disso, oportuniza ao aluno se situar, observar e aplicar de forma criteriosa e reflexiva, princípios e referenciais teórico-práticos que foram apreendidos no decorrer do curso (15).

Percebe-se que a análise documental não indicou a realidade do desenvolvimento curricular, uma vez que a partir dos relatos das coordenadoras de curso foi possível identificar uma abordagem diferenciada ao AIQ em relação àquela presente na grade curricular, demonstrando a presença de um "currículo oculto" que não está explícito, mas que é desenvolvido no decorrer dos cursos de graduação, porém ainda com limitações.

Tendo em vista que toda profissão possui uma cultura e valores que lhes são próprios, o processo de socialização profissional contempla não somente a educação e o treinamento, que acontecem no processo de ensino-aprendizagem, através do currículo formal. Agrega também um "currículo oculto" que envolve, sobretudo, as influências das relações sociais embutidas no processo ensino-aprendizagem, e o aluno é um sujeito ativo e não mero objeto a ser moldado pela instituição (16).

Nesse contexto, os cursos de graduação em enfermagem devem conter um projeto pedagógico, construído coletivamente, colocando o aluno como sujeito central da aprendizagem e o professor como facilitador e mediador do processo ensino-aprendizagem. Este projeto pedagógico deverá buscar a formação integral e adequada do estudante através de uma articulação entre o ensino, a pesquisa e a extensão/assistência (14).

No entanto, diante da necessidade de uma formação generalista do profissional de enfermagem e da complexidade da sistematização do cuidar na atualidade, tem-se a dificuldade na formação de um profissional capacitado de forma integral e específica ao mesmo tempo, para atuar em situações de urgência e emergência. Entretanto, na formação integral do enfermeiro deve-se incluir o conhecimento científico, habilidades e competências essenciais para uma atuação resolutiva e eficaz como integrante articulador da equipe multiprofissional de saúde (17).

Assim, ao se integrar o AIQ na formação do enfermeiro atende-se às diretrizes curriculares estabelecidas para os cursos de enfermagem, que orientam a formação de enfermeiros capazes de conhecer e intervir sobre os problemas e situações de saúde-doença mais prevalentes no perfil epidemiológico nacional, com ênfase na região em que estão inseridos, identificando as dimensões biopsicossociais de seus determinantes (14).

Salienta-se que a prática não se constitui em mera atividade e sim como enfrentamento de eventos, visto que é cada vez mais dependente do conhecimento teórico. A teoria deve, portanto, nortear a orientação dessas práticas, logo é importante a interação e articulação entre elas (18).

Revisão de literatura acerca da formação de enfermeiros apontou os cenários de prática como formas facilitadoras do processo ensino-aprendizagem. Na visão dos estudantes de enfermagem a interlocução entre teoria e prática é a maneira mais significativa para o processo de aprendizagem, sendo que a visualização e aplicação prática do que é ministrado em sala de aula favorece ao acadêmico a compreensão do processo de cuidar (19).

A estrutura dos cursos de graduação em enfermagem deve assegurar a realização de atividades teóricas e práticas que devem estar presentes desde o início do curso, permeando 
toda a formação do enfermeiro, de forma integrada e interdisciplinar (14). Faz-se necessário então a inserção do aluno em campos de prática a fim de complementar a teoria ministrada em sala de aula e de garantir uma formação que contemple aspectos essenciais à prática profissional de enfermagem.

Nesse contexto, o Currículo do Curso de Graduação em Enfermagem deve incluir aspectos complementares de perfil, habilidades, competências e conteúdos, de forma a considerar a inserção institucional do curso, a flexibilidade individual de estudos e os requerimentos, demandas e expectativas de desenvolvimento do setor saúde na região (14).

Dentro dessa perspectiva, a partir de 2001, os ministérios da Saúde e Educação do Brasil têm implementado estratégias de forma articulada para a mudança no processo de ensino-aprendizagem dos cursos de graduação da área da saúde. Ressalta-se a necessidade de que os currículos desses cursos sejam adaptados aos formatos mais atuais, baseados em habilidades gerais e específicas associadas a um perfil pessoal e profissional e uma educação voltada aos principais problemas de saúde da população (20).

A análise documental e as entrevistas realizadas junto às coordenadoras de curso possibilitaram obter uma visão mais completa da inserção do Atendimento Inicial ao Queimado na formação acadêmica dos estudantes de enfermagem da região em estudo. No entanto, ao mesmo tempo esses achados refletem a realidade local, não podendo ser generalizados.

Sugere-se a realização de novos estudos, dentro da perspectiva qualitativa, a fim de identificar como se processa a formação do enfermeiro para atuar frente às vítimas de queimaduras sob o ponto de vista dos estudantes de enfermagem. Busca-se assim fornecer subsídios para ultrapassar os limites para uma formação acadêmica de enfermagem ao mesmo tempo generalista e voltada aos problemas de saúde de forte impacto na população brasileira.

\section{REFERÊNCIAS}

1. Moraes BA, Costa NMS. Understanding the curriculum the light of training guiding health in Brazil. Rev Esc Enferm USP. 2016; 50(Spe): 9-16.

2. Winters JRF, Do Prado ML, Heidemann ITSB. Nursing education oriented to the principles of the Unified Health System: perception of graduates. Esc. Anna Nery. 2016; 20(2): 248-253.

3. Moreira COF, Dias MAS. Diretrizes curriculares na saúde e as mudanças nos modelos de saúde e de educação. ABCS Health Sci 2015; 40(3): 300-305.

4. Fernandes JD, Rebouças LC. Uma década de Diretrizes Curriculares Nacionais para a Graduação em Enfermagem: avanços e desafios. Rev Bras Enferm. 2013; 66(Spe): 95-101.

5. Meschial WC, Oliveira MLF. Initial care to burn victims: nursing students' knowledge - a cross-sectional study. Online braz j nurs [Internet]. Sep 2014 [citado 10 jan 2014]; 13(4): 518-28. Disponível em: http://www.objnursing.uff.br/index. php/nursing/article/view/4744/pdf_325

6. Ministério da Saúde (BR). Brasil. Ministério da Saúde. Portaria GM/MS n.1863, de 29/09/2003. Institui a Política Nacional de Atenção às Urgências. Brasília DF; 2003.

7. Salvador PTCO, Dantas RAN, Dantas DV, Torres GV. The academic education in nursing and multiple-victim incidents: an integrative review. Rev Esc Enferm USP. 2012; 46(3): 742-51.

8. Chaves MM, Brito MJM, Montenegro LC, Alves M. Competencias profesionales de los enfermeros: el método developing a curriculum como posibilidad para elaborar un proyecto pedagógico. Enfermería Global [Internet]. Feb 2010 [citado 02 fev 2014]; 18: 1-18. Disponible en: http://revistas.um.es/eglobal/article/ view/93721/90341 
9. Instituto Brasileiro de Geografia e Estatística (IBGE). Censo demográfico 2010, Paraná [Internet]. Brasilia: IBGE; 2010 [citado 20 jan 2012]. Disponível em: http://www.ibge.gov.br/home/estatistica/ populacao/censo2010/tabelas_pdf/total_ populacao_parana.pdf

10. Ministério da Educação Brasil. Sistema e-MEC de Instituições e Cursos de Educação Superior [Internet]. Brasilia: Ministério da Educação; [citado 26 jun 2012]. Disponível em: http://emec.mec. gov.br/

11. Minayo MCS (org.) Pesquisa social: teoria, método e criatividade. 29 ed. Petrópolis: Vozes; $2010.80 \mathrm{p}$.

12. Wallace HJ, O'Neill TB, Wood FM, Edgar DW, Rea SM. Determinants of burn first aid knowledge: Cross-sectional study. Burns. 2013; 39: 1162-9.

13. Lino MM, Calil AM. O ensino de cuidados críticos/intensivos na formação do enfermeiro: momento para reflexão. Rev Esc Enferm USP. 2008; 42(4): 777-83.

14. Conselho Nacional de Educação. Parecer CNE/CES n. 3, de 7 de novembro de 2001. Dispões sobre as Diretrizes Curriculares Nacionais do Curso de Graduação em Enfermagem [Internet]. Brasília: Ministério da Educação; 2001 [citado 3 jul 2013]. Disponível em: http://portal. mec.gov.br/cne/arquivos/pdf/CES03.pdf
15. Lima TC, Paixão FRC, Cândido EC, Campos CJG, Ceolim MF. Estágio curricular supervisionado: análise da experiência discente. Rev Bras Enferm. 2014; 67(1): 133-140.

16. Finkler M, Caetano JC, Ramos FRS. Ética e valores na formação profissional em saúde: um estudo de caso. Cien Saude Colet. 2013; 18(10): 3033-42.

17. Salvador PTCO, Dantas RAN, Dantas DV, Torres GV. A formação acadêmica de enfermagem e os incidentes com múltiplas vítimas: revisão integrativa. Rev Esc Enferm USP. 2012; 46(3): 742-51.

18. Fernandes JD, Silva RMO, Teixeira GA, Florencio RMS, Silva LS, Rebouças LCC. Aderência de cursos de graduação em enfermagem às diretrizes curriculares nacionais na perspectiva do sistema único de saúde. Esc Anna Nery. 2013; 17(1): 82 $-9$.

19. Canever BP, Prado ML, Backes VMS, Gomes DC. Produção do conhecimento acerca da formação do enfermeiro na América Latina. Rev Gaucha Enferm. 2012; 33(4): 211-220.

20. Menegaz JC, Kloh D, Mattioli Neto H, Martini JG, Ramos FRS, Backes VMS. Reliving the debate concerning nursing education: from where we departed and to where we are going. Invest Educ Enferm. 2013; 31(3): 395-405. 